\title{
Cosmogenic activation study in the Nal(TI) Crystals for COSINE-100 experiment
}

\section{ByungjuPark ${ }^{* \dagger}$}

IBS School, University of Science and Technology (UST), Daejeon 34113, Republic of Korea

E-mail: pbj7363@gmail.com

\begin{abstract}
COSINE-100 is a direct dark-matter (WIMP) search experiment that uses an underground array of low background NaI(TI) scintillating crystals as a target/detector in the Yangyang deep underground laboratory. In spite of the underground experimental environment, the detector array and surrounding materials still contain some radioactive nuclides that were primarily produced by previous exposures to cosmic rays. Studies of the time-dependent contamination levels of these cosmogenic radioactive isotopes are required in order to understand these backgrounds to rare event search experiments. In this presentation, results of studies of cosmogenic nuclide contaminations in the COSINE-100 detector materials and comparisons to MC simulations will be presented.
\end{abstract}

The 39th International Conference on High Energy Physics (ICHEP2018)

4-11 July, 2018

Seoul, Korea

* Speaker.

${ }^{\dagger}$ COSINE-100 Collaborators 


\section{Cosmogenic Isotopes and Summary}

COSINE-100 experiment using eight $\mathrm{NaI}(\mathrm{Tl})$ crystals had underground radioactivity cooling times that ranged from several months to three years, there are still background contributions due to the long-lived cosmogenic isotopes that were activated by cosmic rays while they were on the surface. The major cosmogenic components are ${ }^{22} \mathrm{Na},{ }^{109} \mathrm{Cd},{ }^{125} \mathrm{I}$ and $\mathrm{Te}$ components. Here, we calculated amount of cosmogenic isotopes and compared with MC simulation.

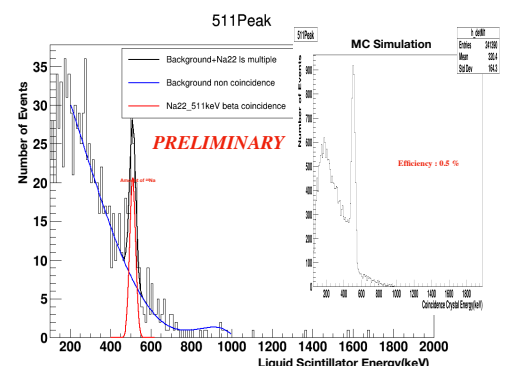

(a)

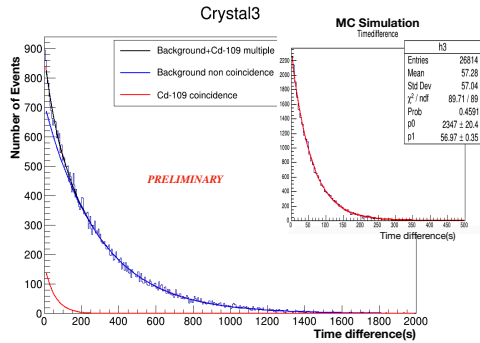

(b)
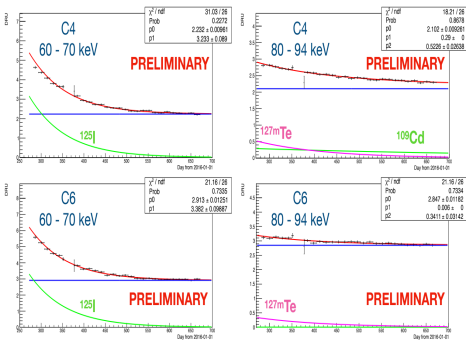

(c)

Figure 1: (a) Triple coincidence events in the same crystal with Double coincidence. Black line is total events and blue line is background evnets and red line is ${ }^{22} \mathrm{Na}$ events. (b) Time difference in Crystal 3. blue line is background events and red line is ${ }^{109} \mathrm{Cd}$ events in the crystal. The meanlife time is $56.29 \mathrm{sec}$ here, it shows red line means ${ }^{109} \mathrm{Cd}$ events. (c)Time difference in crystal 4 and 6 by I and Te component. Green line is ${ }^{125} \mathrm{I}$ and pink line is ${ }^{127 m} \mathrm{Te}$.

Table 1: Summary of COSINE-100 cosmogenic isotopes activity.

\begin{tabular}{l|cccccc}
\hline $\mathrm{I} / \mathrm{C}$ & Crystal1 & Crystal2 & Crystal3 & Crystal4 & Crystal6 & Crystal7 \\
\hline${ }^{3} \mathrm{H}$ & $0.27 \pm 0.040$ & $0.095 \pm 0.031$ & $0.18 \pm 0.056$ & $0.23 \pm 0.050$ & $0.075 \pm 0.040$ & $0.070 \pm 0.032$ \\
${ }^{22} \mathrm{Na}$ & $0.78 \pm 0.18$ & $0.67 \pm 0.16$ & $0.56 \pm 0.11$ & $0.90 \pm 0.15$ & $0.55 \pm 0.14$ & $0.69 \pm 0.19$ \\
${ }^{109} \mathrm{Cd}$ & $0.013 \pm 0.008$ & $0.003 \pm 0.006$ & $0.06 \pm 0.006$ & $0.10 \pm 0.007$ & $0.002 \pm 0.007$ & $0.015 \pm 0.005$ \\
${ }^{125} \mathrm{I}$ & - & - & $0.09 \pm 0.01$ & $0.56 \pm 0.02$ & $0.59 \pm 0.02$ & $0.62 \pm 0.02$ \\
${ }^{121 m} \mathrm{Te}$ & - & - & $0.17 \pm 0.03$ & $0.46 \pm 0.03$ & $0.20 \pm 0.03$ & $0.19 \pm 0.03$ \\
${ }^{125 m} \mathrm{Te}$ & - & - & - & $0.1 \pm 0.1$ & $0.037 \pm 0.12$ & $0.039 \pm 0.11$ \\
${ }^{127 m} \mathrm{Te}$ & - & - & $0.066 \pm 0.012$ & $0.176 \pm 0.009$ & $0.114 \pm 0.011$ & $0.105 \pm 0.011$ \\
\hline
\end{tabular}

\section{References}

[1] E. Komatsu et al. (WMAP Collaboration), Astrophys. J. Suppl. 192 (2011) 18.

[2] R. Bernabei et al., Eur. Phys. J. C 56 (2008) 333.

[3] R. Bernabei et al., Eur. Phys. J. C 67 (2010) 39.

[4] J. Amare et al., Nucl. Instrum. Methods Phys. Res., Sect. A 742 (2014) 187.

[5] Y. D. Kim et al., J. Korean. Phys. Soc. 40 (2002) 520; Y. D. Kim et al., Nucl. Instrum. Methods Phys. Res., Sect. A 552 (2005) 456.

[6] H. S. Lee et al. (KIMS Collaboration), Phys. Lett. B 633 (2006) 201.

[7] S. Agostinelli et al., Nuclear Instruments and Methods A 506 (2003) 250-303. 